\title{
Shape Fitting for the Shape Control System of Silicon Single Crystal Growth
}

\author{
Junli Liang, Miaohua Zhang, Ding Liu and Wenyi Wang
}

\begin{abstract}
Shape fitting, including straight line and ellipse fitting, plays an important role in the (cylinder-) shape control system of silicon single crystal growth because the straight lines and ellipse in the crystal image contain the important horizontal circle center and diameter information. These information can be used as control variables so that the grown crystal approximates to a perfect cylinder and thus can be used as high-quality source materials. In this paper, we develop new straight line and ellipse fitting algorithms. The key points are as follows: i) We formulate the two-dimensional binary image into a singlesnapshot array signal of a virtual sensor array, and casts the angle estimation problem of straight lines into the direction finding one of virtual incoming sources. Based on the virtual array manifold and potential incoming angles, the relevant overcomplete dictionary is constructed and thus a sparse regression problem is formed; ii) To solve such a regression problem, we introduce the weight vector sparsity term into the conventional linear least-squares support vector regression framework to estimate the angles of these straight lines; iii) Based on the estimated angles and potential offsets, another over-complete dictionary is constructed and thus the image can be looked upon as the sparse representation of these dictionary atoms. Since the constructed dictionary is of the same size as the image, we use the compressed sensing theory to reduce the relevant dimensionality and then apply the aforementioned sparse regression method to obtain the relevant offsets of these straight lines; and iv) we derive a new second-order polynomial of ellipse equation to obtained the ellipse parameters to avoid the trival solution from the conventional polynomial model. Some simulation and experimental examples are given to illustrate the effectiveness of the proposed algorithms.
\end{abstract}

Index Terms-Silicon single crystal (SSC), Growth control, Straight line fitting, Ellipse fitting, Least-Squares Support Vector Regression (LS-SVR), Charge-coupled device(CCD) camera.

\section{INTRODUCTION}

I $\mathrm{N}$ the procedure of product manufacture, automatic measurement of product features is one of the most important steps since the timely feedback of these measurements to the relevant control system is helpful to improve the quality of products or to avoid accidents [1]-[5]. In these features, shape information, especially straight line and ellipse (including circle) information [6]-[9], receives lots of attention because it plays an important role in automatic growth of silicon single

This work was supported in part by the National Natural Science Foundation of China under Grants 61471295,61172123 , in part by the Research Youth Star of Shaanxi Province under Grant 2012KJXX-35, and in part by FOK YING TUNG Education Fund under Grant 141119

Junli Liang (Correspondence author), Miaohua Zhang, and Ding Liu are with School of Automation and Information Engineering, Xi' an University of Technology, Xi'an, China, 710048. Email: heery_2004@hotmail.com or liangjunliexaut.edu.cn. Wenyi Wang is with Tianjin Key Lab for Advanced Signal Processing, Civil Aviation University of China,Tianjin,300300,China

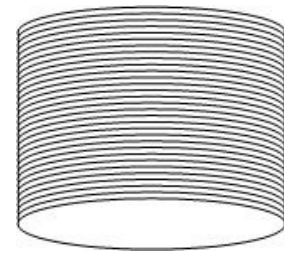

(a)

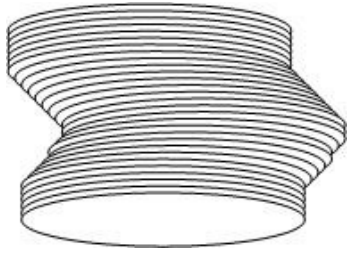

(b)
Fig. 1: Effect of horizontal circle center on crystal growth:(a) perfect cylinder-shape SSC, (b) noncylinder-shape SSC (the crystal slices don't have the same circle center)

crystal (SSC), self-localization and robot orientation of mobile robotics, automatic welding, and vision-based inspection, etc.

In the SSC industry, it is well known that the grown SSC, which approximates a perfect cylinder [9]-[13], can be used as high-quality source materials for semiconductor devices and solar cells, etc. Obviously, such a cylinder-shape control plays an important role in the process of SSC growth. The integral SSC can be looked upon as the crystal slices grown at different times, as shown in Fig. 1 (a). To ensure the grown SSC with the cylinder shape, all the crystal slices must have the same horizontal circle center besides the same diameter. The diameter control technique has received lots of attentions [9]-[11], but the horizontal circle center control holds the equivalent or even more important role. Even if all crystal slices have the same diameter but with different horizontal circle centers, the grown SSC still is not of the cylinder shape, just as shown in Fig. 1(b). More terribly, the disordered crystal slices may touch the pull-crystal furnace wall, resulting in serious accidents. Therefore, it is necessary to obtain both the horizontal circle center and diameter information of the current crystal slice for the shape control system of SSC growth so that this system can adjust the casting speed, furnace temperature, and seeded-head position in time [9], [10]. In the actual application, these information are not detected directly due to the extremely high temperature inside the crystal furnace but from the image acquired by a charge-coupled device (CCD) camera across the viewing window outside the furnace. On the one hand, since the bases of both the CCD camera and the aperture (i.e., a halo with high brightness, which appears at the junction of a solid crystal and a liquid solution) are horizontal, the aperture in the image is a halo of ellipse with zero-angle rotation angle instead of circles due to that the camera is not just above the aperture. The change of aperture diameter is consistent with the diameter of the growing crystal, and thus the aperture diameter can be used as the diameter measurement 
of the currently growing crystal slice. On the other hand, when the growing crystal approximates to a perfect cylinder, the shape edges of the crystal in the image are actually two straight lines, which are not parallel, due to the same camera position reason; otherwise, the shape curves are not straight. Especially, when the current seeded-head position begins to deviate from the correct horizontal circle center, the deviation of the bottom of the shape curves from the straight lines formed by the early grown crystal slices can reflect such a change. Therefore, both the ellipse fitting and straight line fitting plays an important role in the cylinder-shape control system of SSC growth because the horizontal circle center and diameter information mirrored by the ellipses and straight lines can be used as control variables for the relevant control operations so that the grown crystal approximates to a perfect cylinder.

The straight line fitting problem can be described as follows: given a binary image which contains a number of discrete edge points, the objective is to estimate the angles and offsets of these straight lines that fit groups of collinear edge points. Several classical approaches have been proposed for solving this problem [7], [14], [15]. As one of the most excellent line fitting methods, the Hough-transform (HT) method [7] applies a special Radon transform to all points in the image and then accomplishes a two-dimensional search to find the maxima in the angle-offset plane. Another classical algorithm is the Subspace-based LIne DEtection (SLIDE) method [14], [15], which makes an analogy between each line in an image and a planar propagating wavefront radiating on a sensor array [16].

Whereas, the goal of ellipse fitting is to estimate the parameters of an ellipse from a set of co-planar points. The existing fitting algorithms can be classified into two categories: the clustering-like techniques [8] and the least-squares (LS) methods [17]-[20]. The former includes the HT and its variants, which obtain ellipse parameters from the maxima point by carrying out a voting procedure in a multidimensional parameter space due to the fact that an ellipse is specified by multiple parameters. The latter fall into two categories: i) geometric methods, being based on the orthogonal distances between the data points and the estimated ellipse, must solve a nonlinear problem by an iterative procedure [20]. Recently, one of geometric approaches, called the parallel chord method, is developed in [19]. But it will fail when the parallel chords being rigorously perpendicular to a common axis are not available; and ii) algebraic methods, which are extensively used due to their linear nature simplicity and computational efficiency [17], [18]. Especially, the direct least-square fitting (DLSF) method fits an ellipse by solving a generalized eigenvalue problem [17]. Besides the aforementioned methods the recent Bayesian algorithm samples from the joint posterior distribution to obtain ellipse parameters [9].

To fit the straight lines and ellipses in the crystal image and provide accurate measurements for the shape control system of SSC growth, this paper develops new shape fitting algorithms using least-squares support vector regression (LS-SVR) [21], [22]. It is worthwhile to highlight several aspects of the proposed approaches here:

1) we formulate the two-dimensional image into single- snapshot array signals of a virtual sensor array, and casts the angle estimation problem of straight lines into the direction finding one of the virtual incoming sources. Based on the virtual array manifold and potential incoming angles, the relevant over-complete dictionary is constructed and thus a sparse regression problem is formed;

2) To solve such a regression problem, we introduce the weight sparsity term (rather than the conventional socalled Lagrange Multiplier sparsity) into the conventional linear LS-SVR framework to estimate the angles of straight lines;

3) Based on the estimated angels and potential offsets, another over-complete dictionary is constructed and thus the image can be looked upon as the sparse combinations of the dictionary atoms. Since the constructed dictionary is of the same size as the image, we use the compressed sensing theory to reduce the relevant dimensionality and then apply the aforementioned sparse regression method to obtain the relevant offsets of straight lines;

4) we derive a new second-order polynomial model of ellipse equation to obtained the ellipse parameters to avoid the trival solution from the conventional polynomial model.

The rest of this paper is organized as follows. Both problem description and SVR review are given in Section II. The new straight line fitting and ellipse fitting algorithms are developed in Section III. Simulation and experimental results are presented in Section IV. Conclusions are drawn in Section V.

\section{Problem Formulation And LS-SVR ReVieW}

In this section, we firstly formulate the straight line and ellipse fitting problems, then review the classical LS-SVR method.

\section{A. Problem Formulation}

1) Straight Line Fitting: Consider a binary image $\mathbf{D}$ with size $M \times M$, in which only " 1 " and " 0 "-valued pixels are contained. The "1"-valued pixels represent pixels almost collinear with each other in a finite number of groups, and the "O"-valued pixels correspond to the background [7], [14], [15]. For ease of exposition, we take a single line for example, as shown in Fig.2. Each line is characterized by its $\mathrm{x}$-axis offset $\tilde{x}$ and angle $\theta$, and the related equation is given by

$$
x=y \tan \theta+\tilde{x}
$$

The objective of straight line fitting is to estimate the parameters $\left\{\tilde{x}_{1}, \cdots, \tilde{x}_{d}\right\}$ and $\left\{\theta_{1}, \cdots, \theta_{d}\right\}$ of the $d$ straight lines contained in the given image $\mathbf{D}$.

2) Ellipse Fitting: In the crystal image, the aperture ellipse is one with zero-degree rotation angle. Therefore, this paper 


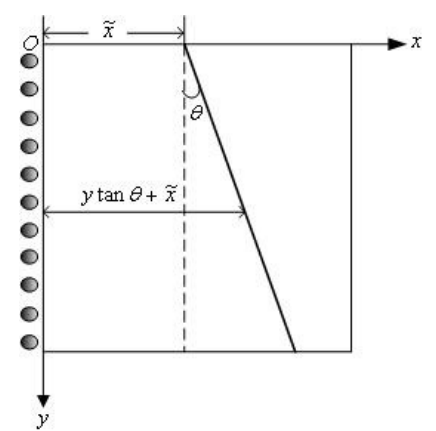

Fig. 2: Straight line contained in an image.

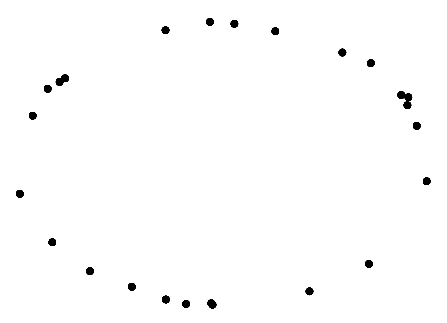

Fig. 3: Data points of an ellipse contained in an image.

focuses on the following ellipse model without rotation angle ${ }^{1}$ (see [9] for details):

$$
\frac{(x-h)^{2}}{r_{x}^{2}}+\frac{(y-k)^{2}}{r_{y}^{2}}=1
$$

where $(h, k)$ denote as the horizontal ellipse center, $\left(r_{x}, r_{y}\right)$ stand for the semi axes in $\mathrm{x}, \mathrm{y}$ coordinates, and $(x, y)$ represent the point on the ellipse, as shown in Fig3. Thus, the objective of ellipse fitting is to estimate the ellipse parameters $\left\{h, k, r_{x}, r_{y}\right\}$ from the given data points $\left\{\left(x_{i}, y_{i}\right)\right\}_{i=1}^{I}$.

\section{B. Review of LS-SVR}

Given a set of training data $\left\{\boldsymbol{x}_{i}, f\left(\boldsymbol{x}_{i}\right)\right\}_{i=1}^{N}$, where $\mathbf{x}_{i}$ and $f\left(\mathbf{x}_{i}\right)$ denote the input vector and output scalar, respectively, then the liner SVR method finds a function

$$
\mathbf{w}^{T} \mathbf{x}+b,
$$

so that $\mathbf{w}^{T} \mathbf{x}_{i}+b$ approximate to $f\left(\mathbf{x}_{i}\right)$ for $i=1, \cdots, N$, where $\mathbf{w}$ and $b$ are the weight vector and bias of the linear SVR model [21], [22], respectively. Especially, the LS-SVR method [22], which receives lots of attentions due to its simplicity resulted from replacing the conventional inequality constraints

\footnotetext{
${ }^{1}$ Although this paper focuses on the zero-degree rotation angle case, its extension to the conventional rotation angle case is straightforward.
}

with equality those, obtains the unknown parameters $\{\mathbf{w}, b\}$ by solving the following problem:

$$
\begin{aligned}
& \min _{\mathbf{w}, b,\{e(i)\}_{i=1}^{N}} \frac{1}{2} \mathbf{w}^{T} \mathbf{w}+\frac{C}{2} \sum_{i=1}^{N} e^{2}(i) \\
& \text { s.t. } f\left(\mathbf{x}_{i}\right)=\mathbf{w}^{T} \mathbf{x}_{i}+b+e(i), \quad i=1, \cdots, N,
\end{aligned}
$$

where $e(i)$ is an error variable and $C$ is the penalty factor in the linear LS-SVR framework.

\section{Proposed Algorithms}

In this subsection, we firstly develop a new straight line fitting algorithm using LS-SVR, and then propose another LSSVR-based method to fit an ellipse.

\section{A. Proposed Straight Line Fitting Algorithm}

It is well known that each straight line is characterized by its angle and offset. In this subsection, we consider estimating the relevant angles and offsets of straight lines in an image consequently.

Define $\mathbf{u}=\left[\begin{array}{ll}1 & e^{j \mu} \cdots e^{j(M-1) \mu}\end{array}\right]^{T}$ using a pre-specified scalar $\mu$, then the binary image $\mathbf{D}$ can be transformed into the following vector form:

$$
\begin{aligned}
\mathbf{z} & =\mathbf{D u} \\
& =\left[\sum_{i=1}^{d} e^{j \mu\left(0 \times \tan \theta_{i}+\tilde{x}_{i}\right)} \sum_{i=1}^{d} e^{j \mu\left(1 \times \tan \theta_{i}+\tilde{x}_{i}\right)} \ldots\right. \\
\left.\sum_{i=1}^{d} e^{j \mu\left((M-1) \times \tan \theta_{i}+\tilde{x}_{i}\right)}\right]^{T} & \\
& =\sum_{i=1}^{d} s_{i} \mathbf{a}\left(\theta_{i}\right)
\end{aligned}
$$

where $s_{i}=e^{j \mu \tilde{x}_{i}}$, and

$$
\mathbf{a}\left(\theta_{i}\right)=\left[\begin{array}{llll}
1 & e^{j \mu \tan \theta_{i}} & e^{j 2 \mu \tan \theta_{i}} & \cdots e^{j(M-1) \mu \tan \theta_{i}}
\end{array}\right]^{T}
$$

From (6), it is easily observed that via the aforementioned transform the binary image $\mathbf{D}$ is transformed into a singlesnapshot array signal $\mathbf{z}$ of a virtual sensor array. More specifically, the virtual array signal $\mathbf{z}$ consists with $d$ virtual incoming source signals with amplitude $s_{i}$ and angle $\theta_{i}$, and the virtual sensor array is a uniform linear array consisted of $M$ sensors [16], [23], just as the solid circles shown in the left part of Fig. 2. Note that $\mu\left|\tan \theta_{i}\right| \leq \pi$ is required to ensure the unique estimation [14], [15].

Unlike the SLIDE method [14], [15], which divided the array signal $\mathbf{z}$ into many snapshots, resulting in low array aperture and inaccurate ( subspace and parameter ) estimates, this paper considers applying the sparse representation technique to estimate $\theta_{i}$ from the single snapshot array signal $\mathbf{z}$ [16].

Let $\left\{\tilde{\theta}_{1}, \tilde{\theta}_{2}, \cdots, \tilde{\theta}_{N_{\theta}}\right\}$ be a sampling grid of all angles of interest within $\left[-90^{\circ}, 90^{\circ}\right]$, and the number of potential angles $N_{\theta}$ is even larger than the number $d$ of incoming array signals. Construct an over-complete dictionary $\mathbf{A}=$ $\left[\mathbf{a}\left(\tilde{\theta}_{1}\right) \quad \mathbf{a}\left(\tilde{\theta}_{2}\right) \cdots \mathbf{a}\left(\tilde{\theta}_{N_{\theta}}\right)\right] \in C^{M \times N_{\theta}}$ [28] where the atom 
$\mathbf{a}\left(\tilde{\theta}_{i}\right)$ has the similar form as that of (6) for $i=1, \cdots, N_{\theta}$. Thus, $\mathbf{z}$ can be represented in another form using $N_{\theta}$ potential signals with amplitudes $\left\{\tilde{s}_{1}, \tilde{s}_{2}, \cdots, \tilde{s}_{N_{\theta}}\right\}$ and angles $\left\{\tilde{\theta}_{1}, \tilde{\theta}_{2}, \cdots, \tilde{\theta}_{N_{\theta}}\right\}$ as:

$$
\mathbf{z}=\mathbf{A} \mathbf{s}+\mathbf{n}
$$

where $\mathbf{s}=\left[\begin{array}{llll}\tilde{s}_{1} & \tilde{s}_{2} \cdots \tilde{s}_{N_{\theta}}\end{array}\right]^{T}$ and $\mathbf{n}$ is the noise. Obviously $\mathbf{s}$ is sparse because when the $i$ th element $\tilde{s}_{i}$ is corresponding to one of the $d$ incoming sources, it is nonzero; otherwise, it equals to zero.

Equation (7) is rewritten in another real-valued form as

$$
\tilde{\mathbf{z}}=\tilde{\mathbf{A}} \tilde{\mathbf{s}}+\tilde{\mathbf{n}}
$$

where

$$
\begin{gathered}
\tilde{\mathbf{z}}=\left[\begin{array}{c}
\operatorname{real}(\mathbf{z}) \\
\operatorname{imag}(\mathbf{z})
\end{array}\right], \\
\tilde{\mathbf{A}}=\left[\begin{array}{cc}
\operatorname{real}(\mathbf{A}) & -\operatorname{imag}(\mathbf{A}) \\
\operatorname{imag}(\mathbf{A}) & \operatorname{real}(\mathbf{A})
\end{array}\right], \tilde{\mathbf{s}}=\left[\begin{array}{c}
\operatorname{real}(\mathbf{s}) \\
\operatorname{imag}(\mathbf{s})
\end{array}\right], \\
\tilde{\mathbf{n}}=\left[\begin{array}{c}
\operatorname{real}(\mathbf{n}) \\
\operatorname{imag}(\mathbf{n})
\end{array}\right],
\end{gathered}
$$

and $\operatorname{real}(\bullet)$ and $\operatorname{imag}(\bullet)$ denote the real and imaginary parts of -, respectively. Actually Equation (8) can be looked upon as a virtual linear system or a linear regression problem, where $\tilde{\mathbf{A}}(i,:)$ represents the virtual input and $\tilde{\mathbf{z}}(i)$ denotes the related output for $i=1, \cdots, 2 M$. Motivated by that LS-SVR can find the parameters with the optimal maximum-margin hyperplane, we apply LS-SVR to obtain $\tilde{\mathbf{s}}$ for the linear regression problem [21], [22]:

$$
\begin{gathered}
\min _{\tilde{\mathbf{s}}, b,\{e(i)\}_{i=1}^{2 M}} \frac{1}{2} \tilde{\mathbf{s}}^{T} \tilde{\mathbf{s}}+\frac{C}{2} \sum_{i=1}^{2 M} e^{2}(i) \\
\text { s.t. } \tilde{\mathbf{z}}(i)=\tilde{\mathbf{A}}(i,:) \tilde{\mathbf{s}}+b+e(i), \quad i=1, \cdots, 2 M .
\end{gathered}
$$

By introducing the Lagrange multipliers $\alpha_{i}$ for $i=$ $1, \cdots, 2 M$, the dual form of (12) can be given by :

$$
\begin{array}{r}
\mathcal{L}(\tilde{\mathbf{s}}, b, e, \boldsymbol{\alpha})=\frac{1}{2} \tilde{\mathbf{s}}^{T} \tilde{\mathbf{s}}+\frac{C}{2} \sum_{i=1}^{2 M} e^{2}(i) \\
+\sum_{i=1}^{2 M} \alpha_{i}\{\tilde{\mathbf{z}}(i)-\tilde{\mathbf{A}}(i,:) \tilde{\mathbf{s}}-b-e(i)\}
\end{array}
$$

The optimality conditions are as follows:

$$
\left\{\begin{array}{l}
\frac{\partial \mathcal{L}}{\partial \tilde{\mathbf{s}}}=0 \rightarrow \tilde{\mathbf{s}}=\sum_{i=1}^{2 M} \alpha_{i} \tilde{\mathbf{A}}(i,:), \\
\frac{\partial \mathcal{L}}{\partial b}=0 \rightarrow \sum_{i=1}^{2 M} \alpha_{i}=0, \\
\frac{\partial \mathcal{L}}{\partial e(i)}=0 \rightarrow \alpha_{i}=C e(i), i=1 \cdots 2 M, \\
\frac{\partial \mathcal{L}}{\partial \alpha_{i}}=0 \rightarrow \tilde{\mathbf{z}}(i)-\tilde{\mathbf{s}}^{T} \tilde{\mathbf{A}}(i,:)-b-e(i)=0, i=1 \cdots 2 M .
\end{array}\right.
$$

According to the Karush-Kuhn-Tucker(KKT) condition, the problem in (13) is reduced to a linear system [22] :

$$
\left[\begin{array}{cc}
\mathbf{Q}+\frac{1}{C} \mathbf{I}_{2 M} & \mathbf{1}_{2 M \times 1} \\
\mathbf{1}_{1 \times 2 M} & 0
\end{array}\right]\left[\begin{array}{c}
\boldsymbol{\alpha} \\
b
\end{array}\right]=\left[\begin{array}{c}
\tilde{\mathbf{z}} \\
0
\end{array}\right],
$$

where $\mathbf{Q}=\tilde{\mathbf{A}} \tilde{\mathbf{A}}^{T}$ and $\boldsymbol{\alpha}=\left[\begin{array}{llll}\alpha_{1} & \alpha_{2} & \cdots & \alpha_{2 M}\end{array}\right]^{T}$. Additionally, $\mathbf{1}_{2 M \times 1}$ denotes a $2 M \times 1$ vector with all elements 1 . Once $\boldsymbol{\alpha}$ and $b$ are solved from (15), the vector $\tilde{\mathbf{s}}$ can be given by

$$
\tilde{\mathbf{s}}=\sum_{i=1}^{2 M} \alpha_{i} \tilde{\mathbf{A}}^{T}(i,:)=\tilde{\mathbf{A}}^{T} \boldsymbol{\alpha}
$$

Although LS-SVR can obtain sparse Lagrange Multipliers [22], it cannot get satisfactory solutions to the aforementioned angle estimation problem of straight lines due to that such a regression problem is related to the sparsity of the weight vector $\tilde{\mathbf{s}}$ rather than the conventional Lagrange Multiplier vector $\boldsymbol{\alpha}$.

To obtain the sparse vector $\tilde{\mathbf{s}}$, we will introduce the sparsity term on $\tilde{\mathbf{s}}$ into the conventional SVR model and construct a new model, which is quite different from the conventional sparsity of Lagrange multipliers $\boldsymbol{\alpha}$ [22]. Note that the equations of $\boldsymbol{\alpha}$ and $b$ in (15) has a unique solution due to the full rank property of $\left[\begin{array}{cc}\mathbf{Q}+\frac{1}{C} \mathbf{I}_{2 M} & \mathbf{1}_{2 M \times 1} \\ \mathbf{1}_{1 \times 2 M} & 0\end{array}\right]$. It is not difficult to prove that because of the full rank property Equation (15) is equivalent to the following minimization problem under a linear constraint:

$$
\begin{aligned}
& \min _{\boldsymbol{\alpha}, b}\left\|\tilde{\mathbf{z}}-\left[\begin{array}{ll}
\mathbf{Q}+\frac{1}{C} \mathbf{I}_{2 M} & \mathbf{1}_{2 M \times 1}
\end{array}\right]\left[\begin{array}{c}
\boldsymbol{\alpha} \\
b
\end{array}\right]\right\|_{2}^{2} \\
& \text { s.t. } \mathbf{1}_{1 \times 2 M} \boldsymbol{\alpha}=0
\end{aligned}
$$

To ensure that $\tilde{\mathbf{s}}$ is sparse, we introduce the sparse constraint term on $\tilde{\mathbf{s}}$ into (17):

$$
\begin{gathered}
\min _{\boldsymbol{\alpha}, b}\left\|\tilde{\mathbf{z}}-\left[\begin{array}{ll}
\mathbf{Q}+\frac{1}{C} \mathbf{I}_{2 M} & \mathbf{1}_{2 M \times 1}
\end{array}\right]\left[\begin{array}{c}
\boldsymbol{\alpha} \\
b
\end{array}\right]\right\|_{2}^{2} \\
+\lambda \sum_{i=1}^{N_{\theta}} \sqrt{\left(\tilde{\mathbf{A}}^{T}(:, i) \boldsymbol{\alpha}\right)^{2}+\left(\tilde{\mathbf{A}}^{T}\left(:, i+N_{\theta}\right) \boldsymbol{\alpha}\right)^{2}} \\
\text { s.t. } \mathbf{1}_{1 \times 2 M} \boldsymbol{\alpha}=0
\end{gathered}
$$

where the parameter $\lambda$ controls the tradeoff between the sparsity of $\tilde{\mathbf{s}}$ and the residual norm $\left\|\tilde{\mathbf{z}}-\left[\begin{array}{ll}\mathbf{Q}+\frac{1}{C} \mathbf{I}_{2 M} & \mathbf{1}_{2 M \times 1}\end{array}\right]\left[\begin{array}{c}\boldsymbol{\alpha} \\ b\end{array}\right]\right\|_{2}^{2}$.

Introducing $N_{\theta}+1$ new variables $\left(\gamma_{1}, \gamma_{2}, \cdots, \gamma_{N_{\theta}}, \eta\right)$, we re-write (18) in another form as:

$$
\begin{gathered}
\min _{\eta, b, \boldsymbol{\alpha}, \gamma_{1}, \cdots, \gamma_{N_{\theta}}} \eta+\lambda \sum_{i=1}^{N_{\theta}} \gamma_{i} \\
\text { s.t. }\left\|\tilde{\mathbf{z}}-\left[\mathbf{Q}+\frac{1}{C} \mathbf{I}_{2 M} \quad \mathbf{1}_{2 M \times 1}\right]\left[\begin{array}{c}
\boldsymbol{\alpha} \\
b
\end{array}\right]\right\|_{2}^{2} \leq \eta, \\
\left\|\left[\begin{array}{c}
\tilde{\mathbf{A}}^{T}(:, i) \\
\tilde{\mathbf{A}}^{T}\left(:, i+N_{\theta}\right)
\end{array}\right] \boldsymbol{\alpha}\right\|_{2} \leq \gamma_{i}, i=1,2, \cdots, N_{\theta}, \\
\mathbf{1}_{1 \times 2 M} \boldsymbol{\alpha}=0 .
\end{gathered}
$$

Equation (19) is easily derived as a standard Second-Order Cone Programming (SOCP) problem and solved using the well-established interior-point methods efficiently [24], [25].

Based on $\tilde{\mathbf{s}}$, we compute the virtual spectrum of array 
signals $\mathbf{p}_{\text {spec }} \in R^{1 \times N_{\theta}}$,the $i$ th element of which is defined as follows:

$$
\mathbf{p}_{\mathrm{spec}}(i)=\sqrt{\tilde{\mathbf{s}}^{2}(i)+\tilde{\mathbf{s}}^{2}\left(i+N_{\theta}\right)}
$$

Thus, the $d$ highest peaks of the spectrum $\mathbf{p}_{\text {spec }}$ indicate the estimated angles $\left(\tilde{\theta}_{1}, \tilde{\theta}_{2}, \cdots, \tilde{\theta}_{d}\right)$.

Once the angles of straight lines are obtained, the remaining task is to estimate the offsets of straight lines. It is assumed the offsets of interest (OI) are $\left\{\tilde{x}_{i, 1}, \tilde{x}_{i, 2}, \cdots, \tilde{x}_{i, N_{i}}\right\}$ related to $\tilde{\theta}_{i}$ for $i=1,2, \cdots, d$. Based on $\tilde{x}_{i, j}$ and $\tilde{\theta}_{i}$, we form a binary image $\mathbf{B}_{i, j}$ with the size $M \times M$, in which only one single straight line with angle $\tilde{\theta}_{i}$ and offset $\tilde{x}_{i, j}$ exists.

Based on $\mathbf{B}_{i, j},(i, j) \in\left[(1,1),(1,2), \cdots,\left(d, N_{d}\right)\right]$, we construct another over-complete dictionary $\mathbf{B}$ with $\sum_{i=1}^{d} N_{i}$ atoms, i.e.,

$$
\mathbf{B}=\left[\operatorname{vec}\left(\mathbf{B}_{1,1}\right) \cdots \operatorname{vec}\left(\mathbf{B}_{d, N_{d}}\right)\right] \in R^{M^{2} \times \sum_{i=1}^{d} N_{i}},
$$

where the atom $\operatorname{vec}\left(\mathbf{B}_{i, j}\right)$ denotes the lexicographically form of $\mathbf{B}_{i, j}$.

Obviously $\operatorname{vec}(\mathbf{D})$ can be represented as a linear combination of $\operatorname{vec}\left(\mathbf{B}_{i, j}\right)$,i.e.,

$$
\mathbf{B v}=\operatorname{vec}(\mathbf{D}),
$$

where the vector $\mathbf{v}$ consists of the combination coefficients and is sparse.

Note that Eq. (22) has the similar form as that of (8). Therefore, it can be solved using Eq.(12)-(19). Note that $\mathbf{B} \in R^{M^{2} \times \sum_{i=1}^{d} N_{i}}$, i.e., the row number of $\mathbf{B}$ is extremely large. To avoid such a "dimension disaster" problem, we apply the compressed sensing theory for dimensionality reduction [26], [27], i.e., define a projection matrix $\phi \in R^{q \times M^{2}}$ to multiply both sides of (22) and obtain another equivalent equation as follows:

$$
\tilde{\mathbf{B}} \mathbf{v}=\tilde{\mathbf{D}},
$$

where $q \ll M^{2}, \tilde{\mathbf{B}}=\boldsymbol{\phi} \mathbf{B} \in R^{q \times \sum_{i=1}^{d} N_{i}}$, and $\tilde{\mathbf{D}}=\boldsymbol{\phi} v e c(\mathbf{D}) \in$ $R^{q \times 1}$.In practical application $\tilde{\mathbf{B}}$ may be selected from the predefined atom sets.

Then, optimization steps similar to Eq.(12)-(19) are applied to solve (23). Once $\mathbf{v}$ is obtained from Eq.(23), the $d$ highest peaks of the absolute values of $\mathbf{v}$ indicate the estimated offsets.

\section{B. Proposed Ellipse Fitting Algorithm}

The standard second-order polynomial form of Eq. (2) is given by:

$$
\begin{aligned}
& \frac{1}{r_{x}^{2}} x^{2}-\frac{2 h}{r_{x}^{2}} x+\frac{1}{r_{y}^{2}} y^{2}-\frac{2 k}{r_{y}^{2}} y+\frac{h^{2}}{r_{x}^{2}}+\frac{k^{2}}{r_{y}^{2}}=1 \\
\Rightarrow & \mathbf{w}^{T} \mathbf{x}+b=1
\end{aligned}
$$

where $\mathbf{x}=\left[\begin{array}{llll}x^{2} & -2 x & -2 y & y^{2}\end{array}\right]^{T}, \mathbf{w}=\left[\begin{array}{llll}\frac{1}{r_{x}^{2}} & \frac{h}{r_{x}^{2}} & \frac{k}{r_{y}^{2}} & \frac{1}{r_{y}^{2}}\end{array}\right]^{T}$, and $b=\frac{h^{2}}{r_{x}^{2}}+\frac{k^{2}}{r_{y}^{2}}$. If we apply (13)-(15) to (24) directly, a useless solution $\mathbf{w}=0$ and $b=1$ will be resulted in. In order to get an efficient solution, we multiply both sides of (24) by $r_{x}^{2}$ to obtain another second-order polynomial:

$$
\begin{aligned}
& (2 x) h+\left(-y^{2}\right) \frac{r_{x}^{2}}{r_{y}^{2}}+(2 y) \frac{r_{x}^{2}}{r_{y}^{2}} k+r_{x}^{2}-h^{2}-\frac{r_{x}^{2}}{r_{y}^{2}} k^{2}=x^{2} \\
& \Rightarrow \mathbf{w}^{T} \mathbf{x}+b=x^{2}
\end{aligned}
$$

where $\mathbf{x}=\left[\begin{array}{lll}2 x & -y^{2} & 2 y\end{array}\right]^{T}, \mathbf{w}=\left[\begin{array}{lll}h & \frac{r_{x}^{2}}{r_{y}^{2}} & \frac{r_{x}^{2}}{r_{y}^{2}} k\end{array}\right]^{T}$, and $b=$ $r_{x}^{2}-h^{2}-\frac{r_{x}^{2}}{r_{x}^{2}} k^{2}$. Obviously, applying the standard LS-SVR method to $(25)$ will yield a reasonable solution, from which the ellipse parameters can be readily obtained.

\section{Discussion}

In this subsection, we analyze the computational complexity of the proposed method and the HT method, which is traditional used in the shape control system of SSC growth [10][11]. Here we take an image of size $M \times M$ for example. The HT method requires $O\left(M^{3}\right)$ to detect lines in such an $M \times M$-dimensional image [8]. However, the proposed straight line fitting method requires $\sqrt{N_{\theta}+2}$ SOCP iterations at most to determine the related angles [24][25], where $N_{\theta}+2$ is the number of the second order cones. For each iteration, it requires $O\left(4 N_{\theta}^{3}\right)$. Besides, the proposed algorithm requires $O\left(4 N_{d}^{3.5}\right)$ to obtain the related offsets [24][25]. Therefore, for the straight line fitting problem, the proposed line fitting method requires $O\left(4 N_{\theta}^{3.5}+4 N_{d}^{3.5}\right)$ at most.

For the ellipse fitting problem, the proposed LS-SVR-based ellipse fitting method requires $O\left(N^{3}\right)$ [29], where $N$ denotes the number of ellipse edge points. However, the HT method requires $O\left(N_{h} \times N_{k} \times N_{r x} \times N_{r y}\right)$ to detect an ellipse (characterized by four parameters, $\left\{h, k, r_{x}, r_{y}\right\}$ ), where $N_{h}$, $N_{k}, N_{r x}$, and $N_{r y}$ denote the divided grids on the horizontal ellipse center ( $\mathrm{x}$ and $\mathrm{y}$ coordinates), and the semi axes ( $\mathrm{x}$ and y directions), respectively.

Commonly $N_{\theta} \ll M$ and $N_{d} \ll M$ hold for the crystal image, and also $N^{3} \ll\left(N_{h} \times N_{k} \times N_{r x} \times N_{r y}\right)$ holds due to the unknown ranges of $\left\{N_{h}, N_{k}, N_{r x}, N_{r y}\right\}$ and fine quantization on them (to ensure accuracy). Therefore, the proposed methods have the lower computational complexity than HT for straight line and ellipse fitting.

\section{Simulation And Experimental Results}

In this section, some simulations and experiments are conducted to verify the performance of the proposed methods.

\section{A. Simulations on Straight Line Fitting}

In this subsection, four simulation examples are implemented by the proposed straight line fitting method. For comparison purpose, the classical HT and SLIDE approaches [7], [15] are also run on the same data. 


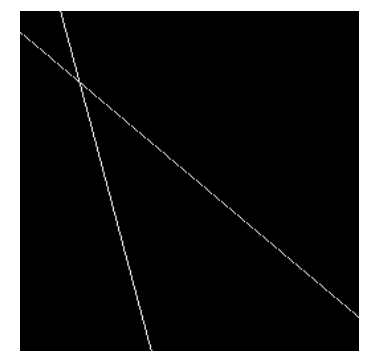

Fig. 4: Two cross lines in Subsec. A.

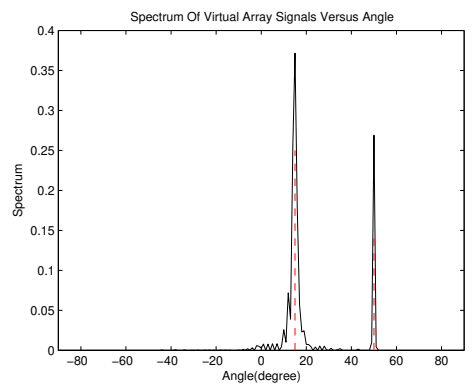

Fig. 5: Angle spectrum for cross lines in Subsec. A.

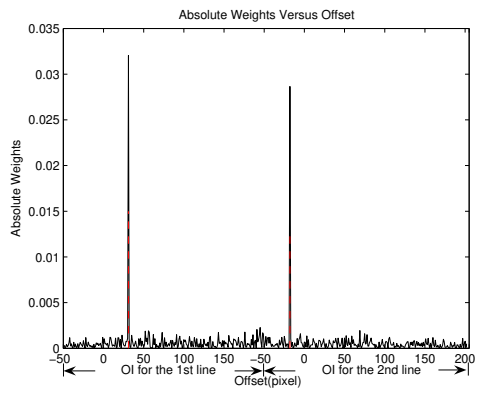

Fig. 6: Absolute values of $\mathbf{v}$ for cross lines in Subsec. A.

1) Two Cross Line Case: In this simulation example, we consider a $256 \times 256$-dimensional image with two cross lines, as shown in Fig.4, which are characterized $\operatorname{by}\left(\theta_{1}=15^{\circ}, \tilde{x}_{1}=30\right)$, and $\left(\theta_{2}=50^{\circ}, \tilde{x}_{2}=-20\right)$, respectively. Based on the image, $\mu=0.6$ is applied to yield a virtual single-snapshot sensor array signal, which consists of two incoming sources. The sampling grid is uniform with $1^{\circ}$ in the range $\left[-90^{\circ}, 90^{\circ}\right]$ i.e., $N_{\theta}=181$. Fig. 5 plots the spectrum obtained by the proposed straight line fitting algorithm with $C=200$ and $\lambda=50$, the two highest peaks of which indicate the estimated angles $\left(\tilde{\theta}_{1}, \tilde{\theta}_{2}\right)$. In addition, let the sampling grid of offsets be uniform with 1 pixel in the range $[-50,200]$. Based on the estimated angels $\left(\tilde{\theta}_{1}, \tilde{\theta}_{2}\right)$ and OI $[-50,200]$, we construct another over-complete dictionary with 502 atoms and then reduce the related atoms and measurement to be $q=800$-dimensional. Fig. 6 plots the absolute values of $\mathbf{v}$ versus offset. The true parameter

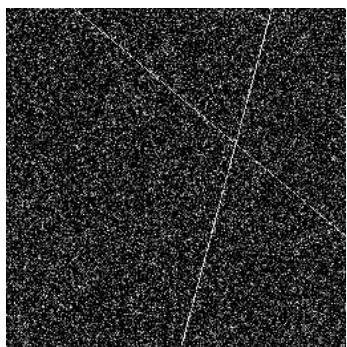

(a)

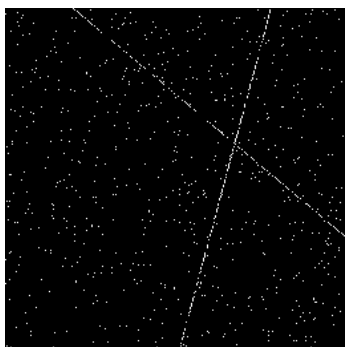

(b)
Fig. 7: (a) an image with Gaussian noise(zeros-mean and standard deviation $\sigma=$ 100),(b) the binary image from threshold segmentation (Threshold value 225).

values are marked in red in Figs. 5 and 6, which shows that the proposed algorithm can estimate the angles and offsets of the two cross lines accurately. For comparison, the HT and SLIDE methods are also implemented, and the related estimation results are given in Table I, which shows that the proposed algorithm has the same performance as HT, and is an alternative straight line fitting method.

TABLE I: Estimation results with cross lines in Subsec. A

\begin{tabular}{|l|l|l|l|l|}
\hline Method & $\tilde{\theta}_{1}$ & $\hat{\tilde{x}}_{1}$ & $\tilde{\theta}_{2}$ & $\hat{\tilde{x}}_{2}$ \\
\hline Proposed & $15^{\mathrm{o}}$ & 30 & $50^{\mathrm{o}}$ & -20 \\
\hline HT & $15^{\mathrm{o}}$ & 30 & $50^{\mathrm{o}}$ & -20 \\
\hline SLIDE & $15.16^{\mathrm{o}}$ & 31.07 & $50.03^{\mathrm{o}}$ & -19.11 \\
\hline
\end{tabular}

2) Two Cross Line With noise: The effect of noise on the performance of the proposed algorithm is investigated in this simulation example. Two cross line corrupted by zerosmean white and homogeneous Gaussian noise are shown in Fig7(a),each line is characterized by $\left(\theta_{1}=-15^{\circ}, \tilde{x}_{1}=200\right)$ and $\left(\theta_{2}=50^{\circ}, \tilde{x}_{2}=50\right)$,respectively. The sampling grid of offsets is uniform with 1 pixel in the range $[50,250]$. Other parameters are the same as Example 1. After threshold segmentation, a binary image is obtained in Fig7(b).The HT method ,SLIDE method and the proposed method are used to estimate the angles and offsets of the lines in the resulted binary image. From the results of the three algorithms listed in Table II, it can be seen that the SLIDE algorithm performs poor under this case while the proposed method and the HT method still perform well.As shown in Figs.8 and 9, the proposed algorithm can detect the angles and offsets of the two lines accurately.

TABLE II: Estimation results of cross lines with noise in Subsec. A

\begin{tabular}{|l|l|l|l|l|}
\hline Method & $\tilde{\theta}_{1}$ & $\hat{\tilde{x}}_{1}$ & $\tilde{\theta}_{2}$ & $\hat{\tilde{x}}_{2}$ \\
\hline Proposed & $-15^{\mathrm{o}}$ & 200 & $50^{\mathrm{o}}$ & 50 \\
\hline HT & $-15^{\mathrm{o}}$ & 200 & $50^{\mathrm{o}}$ & 50 \\
\hline SLIDE & $-14.47^{\mathrm{o}}$ & 202.96 & $50.93^{\mathrm{o}}$ & 42.74 \\
\hline
\end{tabular}

3) Parallel Line Case: This simulation example explores the capability of the proposed algorithm to detect parallel lines, which is quite difficult in the multiple straight line fitting problem. In Fig. 10, there are three lines with the same angle $\theta=-20^{\circ}$ but different offsets $\left(\tilde{x}_{1}=80, \tilde{x}_{2}=90, \tilde{x}_{3}=110\right)$. 


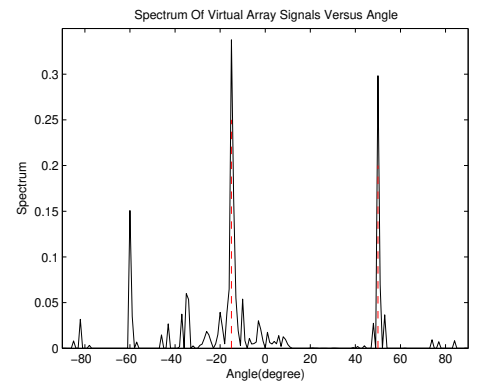

Fig. 8: Angle spectrum for noisy cross lines in Subsec. A.

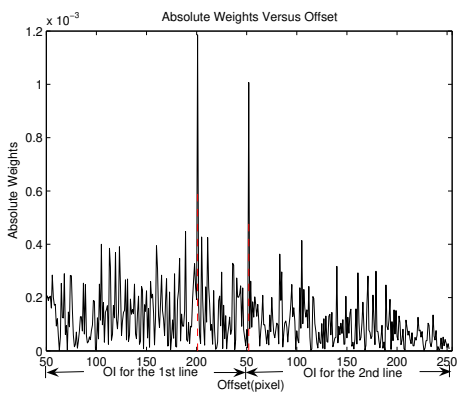

Fig. 9: Absolute values of $\mathbf{v}$ for noisy cross lines in Subsec. A.

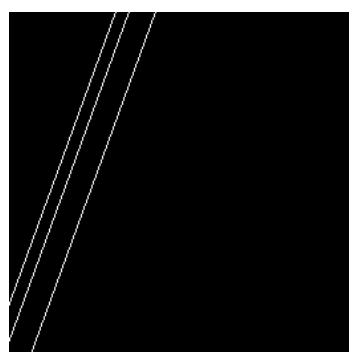

Fig. 10: Parallel lines in Subsec. A

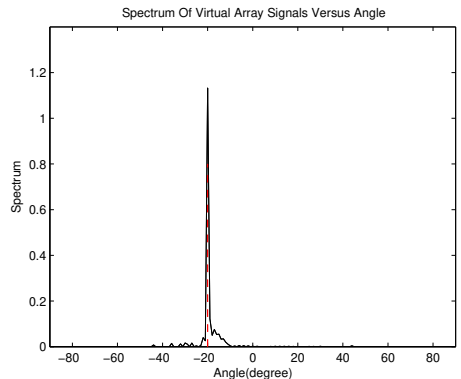

Fig. 11: Angle spectrum for parallel lines in Subsec. A.

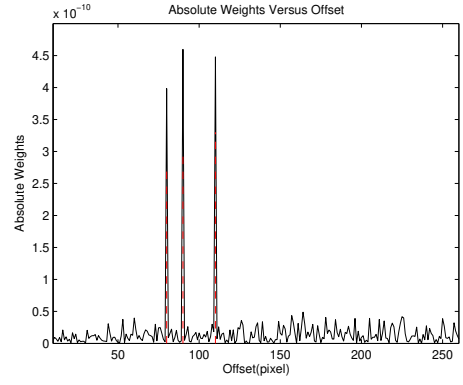

Fig. 12: Absolute values of $\mathbf{v}$ for parallel lines in Subsec. A.

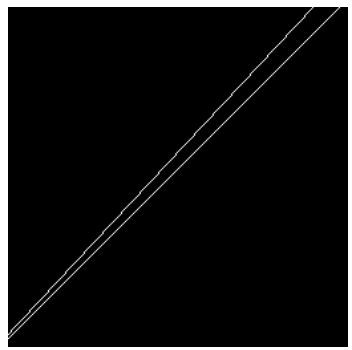

Fig. 13: Lines with small angular gap in Subsec. A.

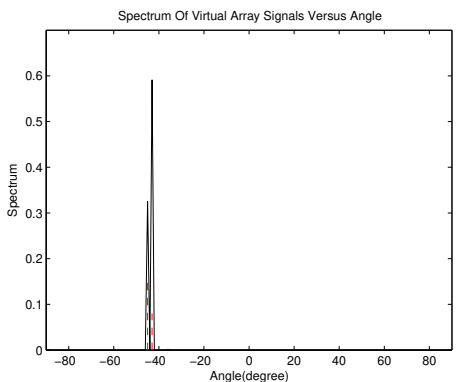

Fig. 14: Angle spectrum for small-gap lines in Subsec. A.

The spectrum of angles and the absolute weights of offsets using the proposed algorithm are given in Figs. 11 and 12, respectively. The estimation results using all the three algorithms are listed in Table III, which show that the proposed algorithm can distinguish parallel straight lines well and is more accurate than SLIDE.

TABLE III: Estimation results under parallel line case in Subsec. A

\begin{tabular}{|l|l|l|l|l|}
\hline Method & $\tilde{\theta}$ & $\hat{\tilde{x}}_{1}$ & $\hat{\tilde{x}}_{2}$ & $\hat{\tilde{x}}_{3}$ \\
\hline Proposed & $-20^{\circ}$ & 80 & 90 & 110 \\
\hline HT & $-20^{\circ}$ & 80 & 90 & 110 \\
\hline SLIDE & $-20.07^{\mathrm{o}}$ & 83.64 & -304.83 & 108.87 \\
\hline
\end{tabular}

4) Small Angular Gap Case: In this simulation example, the effect of small angular gap on the performance of the proposed algorithm is investigated. There are two lines with $\left(\theta_{1}=-43^{\circ}, \tilde{x}_{1}=230\right)$ and $\left(\theta_{1}=-45^{\circ}, \tilde{x}_{1}=250\right)$, as shown in Fig. 13. Figs. 14 and 15 show the experimental results of the proposed algorithm, and Table IV lists the estimation results from all algorithms, which show that the proposed algorithm can estimate the parameter of the straight lines with small angular gap accurately. 


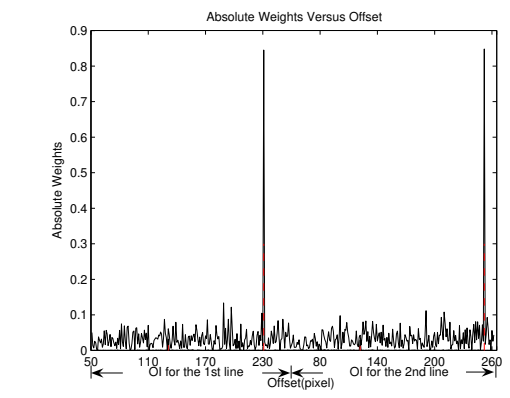

Fig. 15: Absolute values of $\mathbf{v}$ for small-gap lines in Subsec. A.

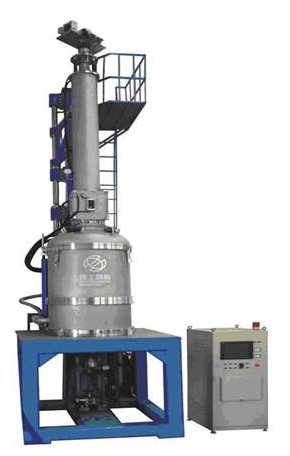

Fig. 16: TDR-150 CZ crystal furnace.

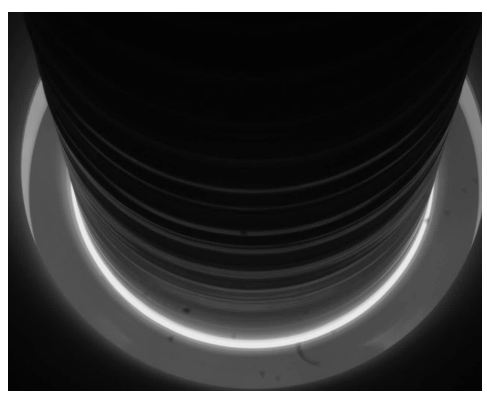

Fig. 17: Crystal image obtained from the CCD camera in Subsec. B.

TABLE IV: Estimation results for small-gap case in Subsec. A.

\begin{tabular}{|l|l|l|l|l|}
\hline Method & $\tilde{\theta}_{1}$ & $\hat{\tilde{x}}_{1}$ & $\tilde{\theta}_{2}$ & $\hat{\tilde{x}}_{2}$ \\
\hline Proposed & $-43^{\mathrm{o}}$ & 230 & $-45^{\mathrm{o}}$ & 250 \\
\hline HT & $-43^{\mathrm{o}}$ & 230 & $-45^{\mathrm{o}}$ & 250 \\
\hline SLIDE & $-42.66^{\mathrm{o}}$ & 227.90 & $-45.29^{\mathrm{o}}$ & 249.24 \\
\hline
\end{tabular}

\section{B. Experiment on High-Quality Crystal Image}

To test the effectiveness of the proposed method on real data, we collected the data points from the crystal furnace shown in Fig.16. Fig.17 displays the crystal image $(1024 \times$ 1280-dimension) obtained from the CCD camera, in which we can see that when the growing crystal approximates to a perfect cylinder, the shape curve of crystal approximates to two straight lines and the aperture area is of the ellipse shape.

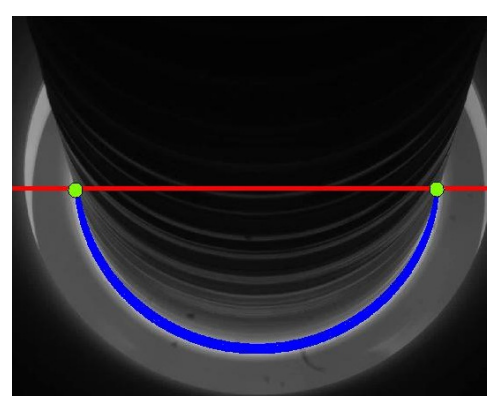

Fig. 18: Region division in Subsec. B.

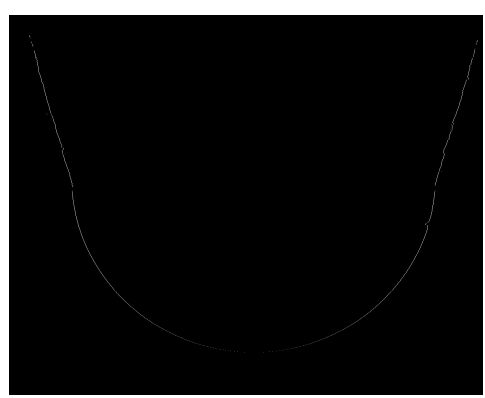

Fig. 19: Edge points of the crystal image in Subsec. B.

To obtain the edges of the shape curves and the aperture, we consider the following preprocessing procedures: i) obtain the aperture area (marked by "blue points" in Fig.18) via threshold segmentation since the aperture area is the brightest region in the entire image; ii) determine the edge points of the aperture area using the Sobel method, then obtain the edge points of outer ellipse via column scan, as shown at the bottom of Fig. 19; iii) obtain the straight line region above the "red line" determined by two "green vertexes" of the aperture area, as shown in Fig.18; and iv) obtain the edge points of the shape curves (i.e., straight lines) by the image enhancement, bright region remove, Prewitt vertical edge detection, and point scan (finding out the edge points with the smallest and largest column indexes for each row) steps, as shown at the top of Fig. 19.

In this experiment, we apply the proposed straight line fitting algorithm to fit the edge points of the crystal curve on the top area of Fig. 19, and fit the edge points of the outer ellipse of aperture using the proposed SVR-based ellipse fitting method. The angle spectrum and the absolute weights of offsets of these lines are given in Fig. 20 and 21, respectively. In addition, the parameter estimation results of straight lines and outer ellipse from all algorithms, including the HT, SLIDE, Bayesian, and DLSF methods [7], [9], [15], [17], are listed in Table V and VI, respectively. A run time on the computer (Intel R, CPU 2.0, 2-GB memory) for all algorithms are also provided in the related tables for comparison purpose.

Fig. 22 displays the fitting results for the crystal image, in which the fitted results of the proposed method and HT method are marked in red (both line and ellipse) and blue (both line 


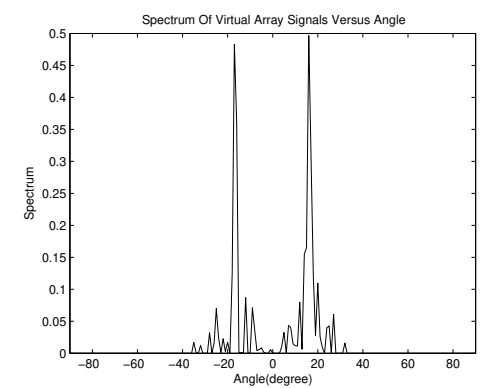

Fig. 20: Angle spectrum for crystal image in Subsec. B.

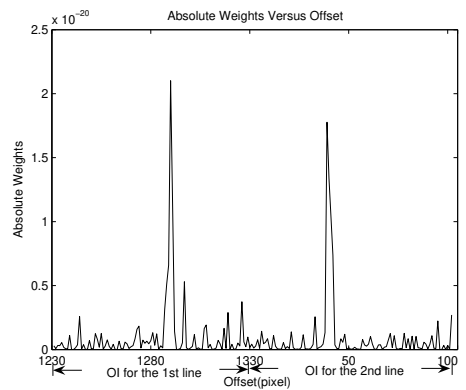

Fig. 21: Absolute values of $\mathbf{v}$ in Subsec. B.

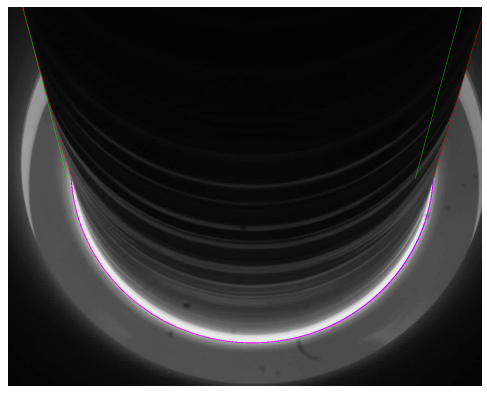

Fig. 22: Fitting results in Subsec. B: the fitted results of the proposed method and HT method are marked in red (both line and ellipse) and blue (both line and ellipse), respectively. In addition, the fitting result from the SLIDE (only line) is marked in green; whereas the results from the Bayesian (only ellipse) and DLSF (only ellipse) methods are marked in cyan and violet, respectively.

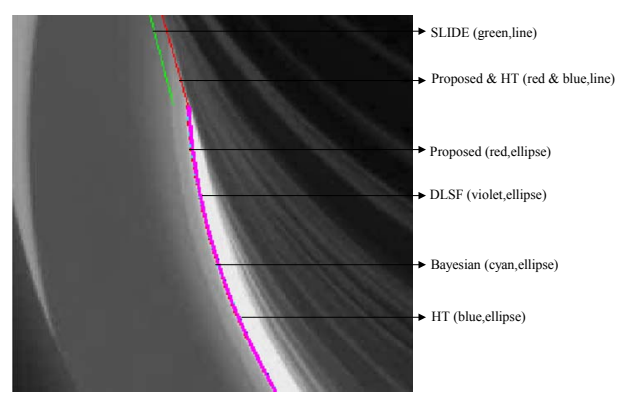

Fig. 23: Magnification of the left local part in Subsec. B.

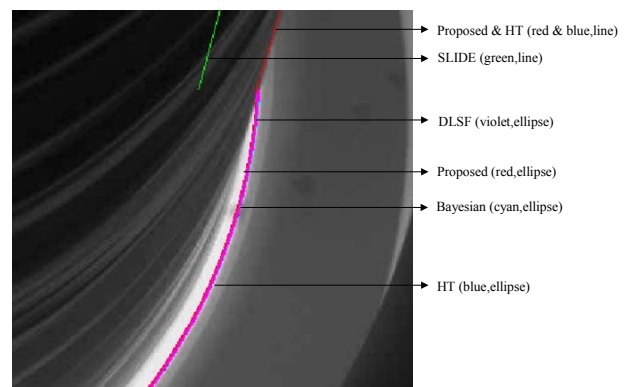

Fig. 24: Magnification of the right local part in Subsec. B.

TABLE V: Estimation results of Crystal data for straight lines in Subsec. B

\begin{tabular}{|l|l|l|l|l|l|}
\hline Method & $\tilde{\theta}_{1}$ & $\tilde{\tilde{x}}_{1}$ & $\tilde{\theta}_{2}$ & $\begin{array}{l}\hat{\tilde{x}}_{2} \text { Run } \\
\text { Time }(\mathrm{s})\end{array}$ & \\
\hline Proposed & $-17^{\mathrm{o}}$ & 1289 & $16^{\mathrm{o}}$ & 37 & 120.24 \\
\hline HT & $-17^{\mathrm{o}}$ & 1289 & $16^{\mathrm{o}}$ & 37 & 219.31 \\
\hline SLIDE & $-15.18^{\mathrm{o}}$ & 1225.24 & $14.51^{\mathrm{o}}$ & 39.54 & 0.53 \\
\hline
\end{tabular}

TABLE VI: Estimation results of Crystal data for outer ellipse in Subsec. B

\begin{tabular}{|l|l|l|l|l|l|}
\hline Method & $\hat{r}_{x}$ & $\hat{r}_{y}$ & $\hat{h}$ & $\hat{k}$ & $\begin{array}{l}\text { Run } \\
\text { Time (s) }\end{array}$ \\
\hline Proposed & 471.30 & 491.14 & 437.95 & 660.67 & 1.37 \\
\hline HT & 472 & 491 & 437 & 660 & 726.84 \\
\hline Bayesian & 472.19 & 491.27 & 437.32 & 660.62 & 16.73 \\
\hline DLSF & 470.27 & 490.81 & 439.01 & 660.58 & 0.09 \\
\hline
\end{tabular}

and ellipse), respectively. In addition, the fitting result from the SLIDE method (only line) is marked in green; whereas the results from the Bayesian (only ellipse) and DLSF (only ellipse) methods are marked in cyan and violet, respectively. We magnify the left and right local parts of Fig. 22 for comparison purpose, as shown in Figs. 23 and 24. From these results, we can see that: i) for the straight line fitting problem, the fitted result of the proposed algorithm overlaps with that of the HT method; whereas the SLIDE method with the least run time exhibits the poorest fitting result, deviating from the true boundary of the crystal; ii)for the ellipse fitting problem, the fitted results from the proposed, HT, Bayesian, and DLSF methods nearly overlap with each other. However, the proposed and DLSF methods require the less run time. Based on the fitting results, the deviation of seeded-head position and the current crystal diameter can be analyzed to implement the related control operations by the shape control system of SSC growth.

\section{Experiment on Poor-Quality Crystal Image}

In this subsection, we test our methods on the poor-quality crystal image $(471 \times 881$-dimension)as shown in Fig. 25, which is noisy, low-resolution, and incomplete (aperture). These shortcomings are not helpful to detect and fit the edge points accurately. Fig.26 displays the corresponding edge points via the above-mentioned preprocessing steps, which are not ideal due to the disadvantageous influence of the factors (noise, low-resolution, and incomplete aperture) on the preprocessing steps. The angle spectrum and the absolute weights of offsets of these lines from the proposed algorithm are given in Fig.27 and Fig.28, respectively. Fig. 29 displays the fitted results from all the aforementioned methods (similar 


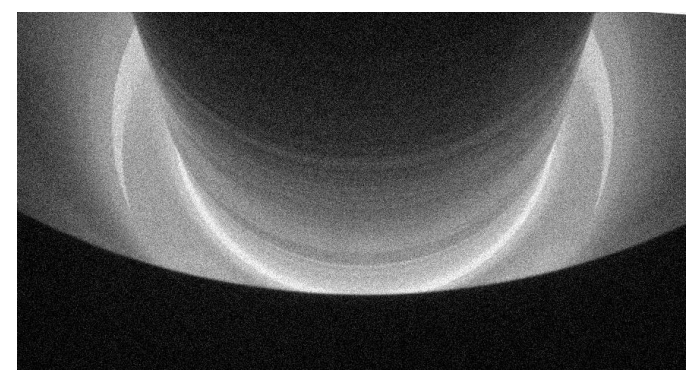

Fig. 25: Poor-quality crystal image in Subsec. C.

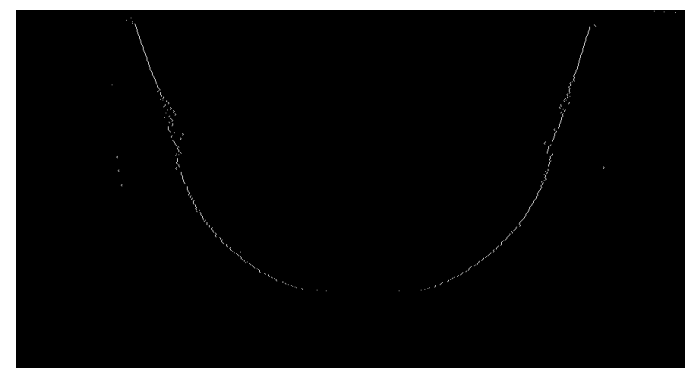

Fig. 26: Edge points in Subsec. C.

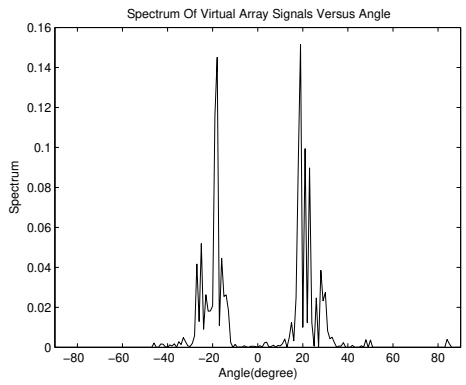

Fig. 27: Angle spectrum in Subsec. C

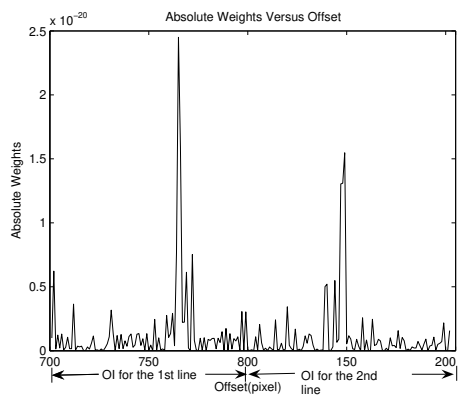

Fig. 28: Absolute values of $\mathbf{v}$ in Subsec. C.

color configurations as Fig. 22 are applied). Additionally, the magnified left and right local parts of Fig. 29 are given in Figs. 30 and 31, respectively. Table VII displays the estimated parameters from the straight line edge points and Table VIII shows the parameter estimates from the ellipse edge points.

From the fitted results, we can see that for the poor-quality crystal image: i)the proposed algorithm and HT method still

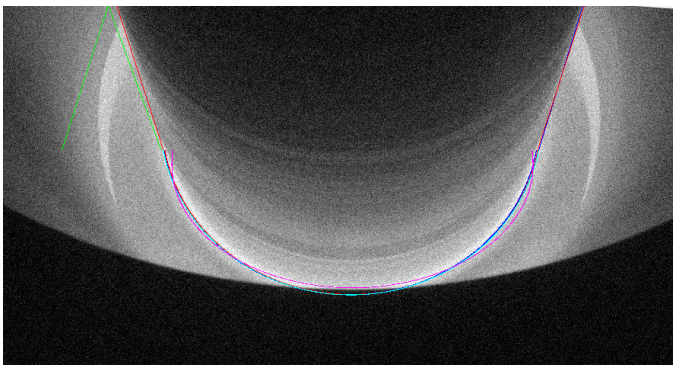

Fig. 29: Fitting results in Subsec. C: the related color configuration is the same as Fig.

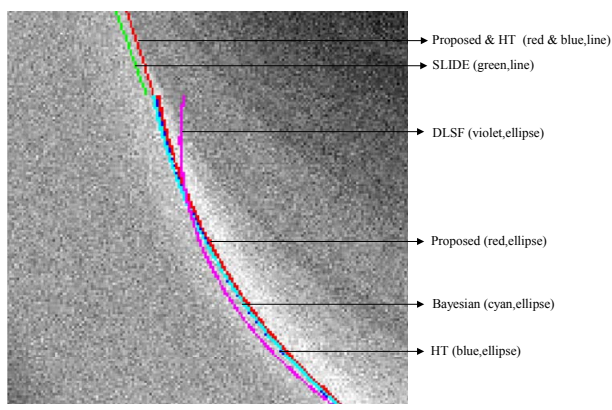

Fig. 30: Magnification of the left local part in Subsec. C.

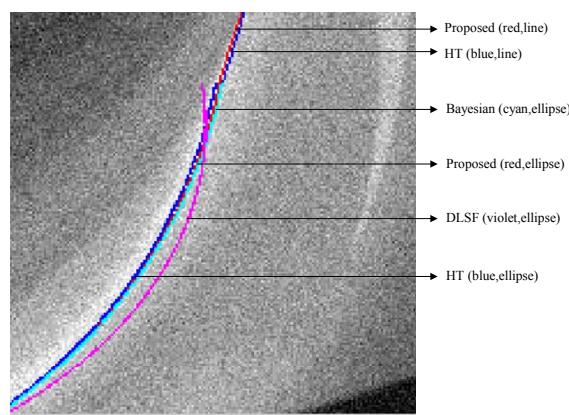

Fig. 31: Magnification of the right local part in Subsec. C.

TABLE VII: Estimation results for straight lines in Subsec. C.

\begin{tabular}{|l|l|l|l|l|l|}
\hline Method & $\tilde{\theta}_{1}$ & $\tilde{\tilde{x}}_{1}$ & $\tilde{\theta}_{2}$ & $\hat{\tilde{x}}_{2}$ & $\begin{array}{l}\text { Run } \\
\text { Time (s) }\end{array}$ \\
\hline Proposed & $-18^{\mathrm{o}}$ & 764 & $18^{\mathrm{o}}$ & 149 & 64.54 \\
\hline HT & $-17^{\mathrm{o}}$ & 762 & $18^{\mathrm{o}}$ & 149 & 73.17 \\
\hline SLIDE & $-17.76^{\mathrm{o}}$ & 137.73 & $20.11^{\mathrm{o}}$ & 138.11 & 0.44 \\
\hline
\end{tabular}

TABLE VIII: Estimation results for outer ellipse in Subsec. C.

\begin{tabular}{|l|l|l|l|l|l|}
\hline Method & $\hat{r}_{x}$ & $\hat{r}_{y}$ & $\hat{h}$ & $\hat{k}$ & $\begin{array}{l}\text { Run } \\
\text { Time (s) }\end{array}$ \\
\hline Proposed & 227.91 & 249.28 & 151.61 & 458.49 & 0.32 \\
\hline HT & 228 & 249 & 152 & 457 & 20.40 \\
\hline Bayesian & 231.18 & 251.27 & 148.91 & 458.03 & 2.68 \\
\hline DLSF & 162.23 & 238.11 & 208.47 & 460.09 & 0.05 \\
\hline
\end{tabular}


work well for the line fitting problem.However, the SLIDE method cannot obtain the satisfactory fitting result; ii)the proposed, HT, and Bayesian methods work well for the ellipse fitting problem. However, the DLSF method with the least run time exhibits the worst fitting result for this case.

The aforementioned experiments, especially the 2 nd and 3rd experiments, show that the proposed algorithms are alternative methods for fitting the line-shape crystal boundary and ellipseshape aperture boundary. Additionally, it is easily found from the 2nd and 3rd experiments that:

1) For the line fitting problem, the SLIDE method requires the least run time, but it exhibits the worst fitting result (not robust), especially when the crystal image suffers from some disadvantages factors such as noise, low-resolution, and incomplete aperture. The proposed straight line fitting method has the approximate (robust) fitting performance as that of HT, but it needs the less run time than HT;

2) For the ellipse fitting problem, the DLSF method requires the least run time, but it exhibits the worst fitting result (not robust) especially when the crystal image is of poor quality. The proposed ellipse fitting method has the approximate performance as those of the HT and Bayesian methods, but it needs the less run time than both of them;

3) The proposed line and ellipse fitting methods have the approximate robust performance as that of HT no matter what the crystal image be of high or poor quality, but it is more efficient than the HT method commonly used in the SSC growth system;

4) The SSC growth process is an extremely slow process. For example, to grow a crystal with length 750-mm, the SSC growth system of TDR-150 CZ crystal furnace requires 80 hours or so ( In other words, in one hour the length of crystal increases by $10 \mathrm{~mm}$ ). Therefore, the run time of the proposed algorithms is sufficient for the crystal growth.

\section{Conclusions}

The SSC shape control is a key step to ensure that the grown crystal is of the cylinder shape and thus can be used as highquality source materials. The straight lines and outer ellipse in the crystal image mirror the variation of the horizontal circle center and the change of the current diameter, respectively, which can be used as efficient control variables for the shape control system of SSC growth. The proposed straight line fitting algorithm introduces the weight vector sparsity term into the LS-SVR framework, and obtains the angles of lines from a virtual single-snapshot array signal. Additionally a similar step is applied to determine the offsets of lines. Furthermore, we apply the conventional LS-SVR method to a new derived second-order polynomial of ellipse equation, yielding the reasonable solution to the ellipse parameters. Experimental results show that the proposed algorithms can fit straight lines and ellipses for the shape control system of SSC growth as well as HT while being more efficient than HT.

\section{REFERENCES}

[1] L. Luo, Z. Lin, and X. Lai. New optimal method for complicated assembly curves fitting. The International Journal of Advanced Manufacturing Technology. vol. 21, pp. 896-901, 2003.

[2] H. Y Tseng, and C. C. Lin. A simulated annealing approach for curve fitting in automated manufacturing systems. Journal of Manufacturing Technology. vol. 18, no.2, pp. 202-216, 2007.

[3] R. M. Inigo, E. S. McVey, B. J. Berger, and M. J. Wirtz, Machine vision applied to vehicle guidance, IEEE Trans. Pattern Analysis and Machine Intelligence. vol. 6 ,no. 7, pp. 820-826, 1984.

[4] H. Y. Tseng. Welding parameters optimization for economic design using neural approximation and genetic algorithm. The International Journal of Advanced Manufacturing Technology. vol. 27, nos.9/10, pp.897-901, 2006.

[5] A.A.M. Assidiq, O.O. Khalifa, R. Islam, and S. Khan, Real time lane detection for autonomous vehicles. in International Conference on Computer and Communication Engineering. pp. 82-88, 2008.

[6] A. K. Jain, Fundamentals of Digital Image Processing. Englewood Cliffs, NJ: Prentice-Hall. 1989.

[7] R. O. Duda and P. E. Hart, Use of the Hough transform to detect lines and curves in pictures. Comm. ACM. vol. 15, pp.11-15, 1972.

[8] V. F. Leavers, Shape Detection in Computer Vision Using the Hough Transform. New York: Springer-Verlag, 1992.

[9] D. Liu and J. Liang. A Bayesian approach to diameter estimation in the diameter control system of silicon single crystal growth. IEEE Trans. Instrumentation and Measurement. vol. 60, no.4, pp.1307-1315, 2011.

[10] C. W. Lan. Recent progress of crystal growth modeling and growth control. Chemical Engineering Science. vol. 59, no.7, pp. 1437-1457, 2004.

[11] U. Ekhult and T. Carlberg. Czochralski growth of tin crystals under constant pull rate and IR diameter control. Journal of Crystal Growth. vol. 76,no. 2, pp. 317-322,1986.

[12] D. T. J. Hurle. Control of diameter in Czochralski and related crystal growth techniques. Journal of Crystal Growth. vol. 42, pp. 473-482, 1977.

[13] K. Takagi, T. Ikeda, T. Fukazawa, and M. Ishii. Growth striae in single crystals of gadolinium gallium garnet grown by automatic diameter control.Journal of Crystal Growth. vol. 38, no.2, pp.206-212,1977.

[14] H. K. Aghajan, and T. Kailath. Sensor array processing techniques for super resolution multi-line-fitting and straight edge detection. IEEE Trans. Image Processing. vol.2, no.4, pp. 454-465, 1993.

[15] H. K. Aghajan and T. Kailath. SLIDE: subspace-based line detection. IEEE Trans. Pattern Analysis and Machine Intelligence. vol.16, no.11, pp. 1057-1073, 1994.

[16] J. Liang, D. Liu, Y. Zhao, and N. Song, Multi-line Fitting Using TwoStage Iterative Adaptive Approach. ICIRA2012,vol.1, pp.144-153, 2012.

[17] A. Fitzgibbon, M. Pilu, and R. B. Fisher. Direct least square fitting of ellipse. IEEE Trans. Pattern Analysis and Machine Intelligence. vol. 21, no.5, pp.476-480, 1999.

[18] W. Gander, G. H. Golub, and R. S. Trebel. Least-squares fitting of circles and ellipses. BIT Numerical Mathematics. vol. 34,issue.4, pp.558-578, 1994.

[19] D. S. Barwick. Very fast best-fit circular and elliptical boundaries by chord data. IEEE Trans. Pattern Analysis and Machine Intelligence. vol. 31, no.6, pp.1147-1152, 2009.

[20] S. J. Ahn, W. Rauh, and H.J. Warnecke. Least-squares orthogonal distances fitting of circle, sphere, ellipse, hyperbola, and parabola. Pattern Recognition. vol. 34, no.12, pp.2283-2303, 2001.

[21] J. L. Rojo-Alvarez, G. Camps-Valls, and M. Martinez-Ramon, etc. Support vector machines framework for linear signal processing, Signal Processing. vol.85, no.12, pp. 2316-2326, 2005.

[22] J. A. K. Suykens and J. Vandewalle, Least squares support vector machine classifiers. Neural Process. Lett., vol.9, no.3, pp.293-300, 1999.

[23] Junli Liang and Ding Liu. Passive localization of mixed near-field and far-field sources using two-stage MUSIC algorithm. IEEE Trans. Signal Processing. vol.58, no.1, pp.108-120, 2010.

[24] J. F. Sturm. Using SeDuMi 1.02, a Matlab toolbox for optimization over symmetric cones. Optim Methods Software, vol.11-12, pp. 625653, 1999 .

[25] MS Lobo, L Vandenberghe, S Boyd, H Lebret, applications of secondorder cone programming, Linear algebra and its applications. vol. 184, no.1, pp.193-228, 1998 .

[26] D. L. Donoho. Compressed sensing. IEEE Trans. Information Theory. vol.52, no.4, pp.1289-1306, 2006.

[27] E. J. Candes, and M. B. Wakin. An introduction to compressive 
sampling. IEEE Signal Processing Magazine. vol.25, no.2, pp.21-30, 2008.

[28] I. Tosic, and P. Frossard. Dictionary Learning. IEEE Signal Processing Magazine. vol.28, no.2, pp.27-38, 2011.

[29] T. V. Gestel, J. A. K. Suykens, and B. Baesens, et al. Benchmarking least squares support vector machine classifiers, Machine Learning, vol. 54, no. 1 , pp. $5^{\prime \prime} \mathrm{C} 32,2004$.

Junli Liang was born in China. In 2007, he received his $\mathrm{Ph} . \mathrm{D}$. degree in signal and information processing from the Institute of Acoustics, Chinese Academy of Sciences. So far, he has published 20 papers in the IEEE Trans. Image Processing, IEEE Trans. Signal Processing, IEEE Trans.Industrial Informatics, IEEE Trans. Antennas and Propagation, IEEE Trans. Aerospace and Electronic Systems, IEEE Trans. Instrumentation and Measurement, IEEE Sensors Journal, Signal Processing (Elsevier), and Digital Signal Processing (Elsevier), etc. His current research interests include signal processing, image processing and pattern recognition, etc.

Miaohua Zhang is currently with the School of Automation and Information Engineering, Xi' an University of Technology, Xi' an China. Her current research interests include image processing and pattern recognition.

Ding Liu is currently with the School of Automation and Information Engineering, Xi' an University of Technology, Xi' an China. His current research interests include crystal growth control and digital signal processing.

Wenyi Wang is currently with civil aviation university of China. His current research interests include adaptive signal processing and satellite navigation. 